\title{
Discrimination of healthy and cancer cells of the bladder by metabolic state, based on autofluorescence
}

\author{
Palmer, S. ${ }^{1}$, Litvinova, K. ${ }^{1}$, Rafailov, E.U. ${ }^{2} \&$ Nabi, G. ${ }^{1}$ \\ 1 - Division of Imaging and Technology, University of Dundee, Ninewells Hospital and Medical \\ School, James Arrott Drive, Dundee, UK DD19SY \\ 2 - Photonics and Nanoscience Group, Aston University, Birmingham, UK B47ET
}

\begin{abstract}
Bladder cancer is among the most common cancers worldwide $\left(4^{\text {th }}\right.$ in men). It is responsible for high patient morbidity and displays rapid recurrence and progression. Lack of sensitivity of gold standard techniques (white light cystoscopy, voided urine cytology) means many early treatable cases are missed. The result is a large number of advanced cases of bladder cancer which require extensive treatment and monitoring. For this reason, bladder cancer is the single most expensive cancer to treat on a per patient basis. In recent years, autofluorescence spectroscopy has begun to shed light into disease research. Of particular interest in cancer research are the fluorescent metabolic cofactors NADH and FAD. Early in tumour development, cancer cells often undergo a metabolic shift (the Warburg effect) resulting in increased NADH. The ratio of NADH to FAD ("redox ratio") can therefore be used as an indicator of the metabolic status of cells. Redox ratio measurements have been used to differentiate between healthy and cancer breast cells and to monitor cellular responses to therapies. Here, we have demonstrated, using healthy and bladder cancer cell lines, a statistically significant difference in the redox ratio of bladder cancer cells, indicative of a metabolic shift. To do this we customised a standard flow cytometer to excite and record fluorescence specifically from NADH and FAD, along with a method for automatically calculating the redox ratio of individual cells within large populations. These results could inform the design of novel probes and screening systems for the early detection of bladder cancer.
\end{abstract}

Keywords: Bladder cancer, Cytometry, Metabolism, Redox ratio, Autofluorescence

Photonic Therapeutics and Diagnostics XI, edited by Bernard Choi, et al., Proc. of SPIE Vol. 9303, 93030T · @ 2015 SPIE · CCC code: 1605-7422/15/\$18 · doi: 10.1117/12.2077218 


\section{INTRODUCTION}

Bladder cancer (BCa) is among the most common cancers worldwide, is responsible for significant patient morbidity and is a particular issue in aging populations ${ }^{1}$. It can be broadly split into two categories: muscle invasive bladder cancer (MIBC) and non-muscle invasive bladder cancer (NMIBC). NMIBC is by far the most common form of BCa but if left unchecked can progress to invasive phenotypes. Furthermore, over two-thirds of NMIBC instances will recur. This means it is imperative to monitor patients with the disease very closely after initial diagnosis and/or therapy. Follow-up surveillance of $\mathrm{BCa}$ is achieved using one or both of white light cystoscopy (WLC) and voided urine cytology (VUC). Unfortunately, WLC and VUC, although useful, suffer from relatively low sensitivity, especially for low grade, early stage tumours and CIS $^{2}$. This results in sub-optimal patient surveillance and high levels of $\mathrm{BCa}$ recurrence and progression $^{3}$. The high levels of recurrence and progression in $\mathrm{BCa}$ and the associated treatment and surveillance costs make $\mathrm{BCa}$ among the most expensive cancers to monitor and treat per patient ${ }^{4}$. For this reason, numerous attempts have been made to develop $\mathrm{BCa}$ detection techniques with improved sensitivity. Chief among these was the advent of photodynamic diagnosis (PDD), achieved by applied photosensitising dye (PS) into the bladder and utilising its preferential uptake by tumours as a guide for imaging modalities ${ }^{5}$. PDD shows selective benefits compared to WLC owing to its greatly improved sensitivity, however this comes at the cost of diminished specificity, high cost and increased patient lay-up time ${ }^{6,7}$. VUC is an attractive tool for screening surveillance of bladder cancer owing to its noninvasive nature, however its relative lack of sensitivity and the need for skilled pathologists to interpret results serve as considerable drawbacks. Many commercially available kits have been developed for urine analysis and bladder cancer detection $^{8}$, including the NMP22 test, Urovysion and BTA STAT. Unfortunately, many large scale meta-analyses of these tests have found them to be no more effective than VUC for the detection and surveillance of $\mathrm{BCa}^{9}{ }^{10}$. It is evident that new avenues must therefore be sought for the early detection of BCa. Autofluorescence spectroscopy has enjoyed success in recent years as an imaging modality for detection of numerous cancers (bladder included) $)^{11-14}$. This is a technique which relies on the inherent fluorescence of cells and tissues to document changes in physiology, without the need for dyes. Two diagnostically relevant endogenous fluorophores are reduced Nicotinamide dinucleotide (NADH) and Flavin adenine dinucleotide (FAD), which can be observed to monitor metabolic changes in cells. Early in tumour progression, cancer cells often undergo a metabolic switch to favour energy production through aerobic glycolysis in place of oxidative phosphorylation (known as the Warburg effect ${ }^{15}$ ). The structure and function of the electron transport chain (ETC) - responsible for oxidative phosphorylation - with NADH as the principal electron donor means that ETC shutdown leads to the accumulation of NADH within cells which have undergone a "glycolytic switch". The "optical redox ratio" (ORR) allows us to take advantage of the optical properties of NADH and the related electron acceptor FAD to detect ETC shutdown in cancerous cells. The ORR is calculated as [NADH]/[FAD], with cancer cells showing increased ORR compared to healthy controls. The accuracy of the ORR has previously been demonstrated in precancerous epithelia ${ }^{16,17}$ suggesting its worth as a tool for sensitive monitoring of early stage and grade BCa. Our main aim was to compare the ORR of healthy and BCa cell lines using a customised flow cytometer, to inform potential VUC strategies based on cellular autofluorescence. Our hypothesis was that BCa cells would possess a significantly increased ORR compared to healthy controls owing to ETC shutdown and NADH accumulation.

\section{METHODS}

\section{Cell culture}

HUC bladder urothelial cell line (Caltag Medsystems) and $5637 \mathrm{BCa}$ cell line (ATCC) were grown to confluence in $75 \mathrm{~cm}^{2}$ culture flasks (Corning) with Dulbecco's Modified Eagle Medium (Sigma-Aldrich) including 1\% PenicillinStreptomycin and 10\% Fetal Bovine Serum. Cells were prepared for study by trypsinising according to standard protocol. Cell suspension was passed through a $30 \mu \mathrm{m}$ filter into a FACS tube. Cells were suspended in PBS (1\% FBS) at approximately $4 \times 10^{6} / \mathrm{ml}$ in all cases. All cell lines used, and the working environment, were certified Mycoplasma free. 


\section{Fluorescence Spectroscopy of biomarkers}

NADH and FAD were purchased as powders (Sigma-Aldrich) and prepared to $10 \mu \mathrm{M}$ solutions in sterile PBS to be studied using a 'LAKK-M' laser spectroscopy device (SPE-LAZMA, Moscow, Russia). A 5ml solution was added to a matte black cuvette (SPE-LAZMA, Moscow, Russia). Solutions of NADH and FAD were excited sequentially with UV $(365 \mathrm{~nm})$ and Blue $(450 \mathrm{~nm})$ sources to optimally excite the fluorophores. Data was visualised using custom-built software (SPE-LAZMA).

\section{Confocal microscopy}

"5637" BCa cells were grown in Petri dishes with coverslip thickness bottoms \#1.5 (WillCoWells) in DMEM (10\% FBS, $1 \%$ Pen-Strep), until $75 \%$ confluent. Cells were stained with a 100nM solution of MitoTracker Red (Molecular Probes) for 45 minutes. A Carl Zeiss 710 confocal microscope was used. NADH was excited at 350nm and FAD was excited at $488 \mathrm{~nm}$. Fluorescence was captured using a CCD camera with 450/20 and 530/20 bandpass filters to isolate NADH and FAD fluorescence, respectively. MitoTracker Red was excited at $579 \mathrm{~nm}$ and detected at $599 \mathrm{~nm}$. A total of 50 cells were studied in this manner. The three excitation wavelengths were used sequentially and data stored for each frame. Images of cells captured using CCD were processed using ImageJ (imageJ.nih.gov). Images from all fluorophores were merged to overlap mitochondrial fluorescence and autofluorescence.

\section{Flow cytometry}

BD Fortessa flow cytometer (Becton Dickinson) was used. Both healthy and cancerous cells were passed through the system at "low" speed setting to set voltages and template population gates. Cell data was acquired at high speed from 10,000 events. In-built blue laser $(488 \mathrm{~nm})$ was used at a power setting of $162 \mathrm{~V}$ to analyse forward scatter and signal width of events. All populations of cells were plotted logarithmically with respect to forward scatter and signal width. All samples were gated identically on forward scatter and signal width to remove dead and doublet cells. NADH and FAD were sequentially excited using UV laser $(360 \mathrm{~nm})$ and blue laser $(488 \mathrm{~nm})$, respectively. Excitation sources were kept as close to excitation maxima as possible for the molecules of interest (NADH Ex Max $=350 \mathrm{~nm}$; FAD Ex Max = $450 \mathrm{~nm})$. UV laser was run at a power setting of $211 \mathrm{~V}$, blue laser was run at a power setting of 397V. The NADHspecific signal was processed using a 450/50 bandpass filter (NADH Em Max $=450 \mathrm{~nm}$ ), and the FAD-specific signal was processed using a 530/30 bandpass filter (FAD Em Max 530nm). FCS data files containing all data were saved and transferred for analysis. Compensation was not required as all results were derived as ratios. Cell viability was determined following flow cytometry on both cell types in suspension using trypan blue and confirmed to be over $95 \%$ for all samples.

\section{Data analysis}

FCS data files were analysed using FCS Express Flow Cytometry Package. Bivariate dot plots were generated on a logarithmic scale based on forward scatter and signal width of each event. An inclusive elliptical gate was drawn between co-ordinates $3.8 \mathrm{~K}, 68 \mathrm{~K}$ and $113 \mathrm{~K}, 154 \mathrm{~K}$. The percentage of cells gated in for each sample were as follows: HUC sample 1 - 85.9\%; HUC sample 2-68.3\%; HUC sample $3-72 \%$; 5637 sample 1 - 89.3\%; 5637 sample 2 91.5\%; 5637 sample $3-71.8 \%$. Individual data on NADH and FAD data was exported from FCS Express, following gating, to Origin Pro 8 statistics software, to calculate means, medians, standard deviations, and standard errors. The average redox ratio was calculated for each population by dividing average NADH fluorescence across $400-500 \mathrm{~nm}$ by average FAD fluorescence across 500-560nm to yield a real number between $0-10$. Average redox ratios of whole populations of cells were analysed for significant differences using student's $t$ test. 


\section{RESULTS}

\section{Fluorescence spectra of NADH and FAD}

NADH possesses fluorescence maximum at 490nm (Fig.2a). FAD has its maximum at 550nm (Fig.2a\&b). NADH displays no fluorescence upon 450nm excitation (Fig.2b); however, there is a significant degree of FAD fluorescence when excited at 365nm (Fig.2a), which overlaps with NADH.
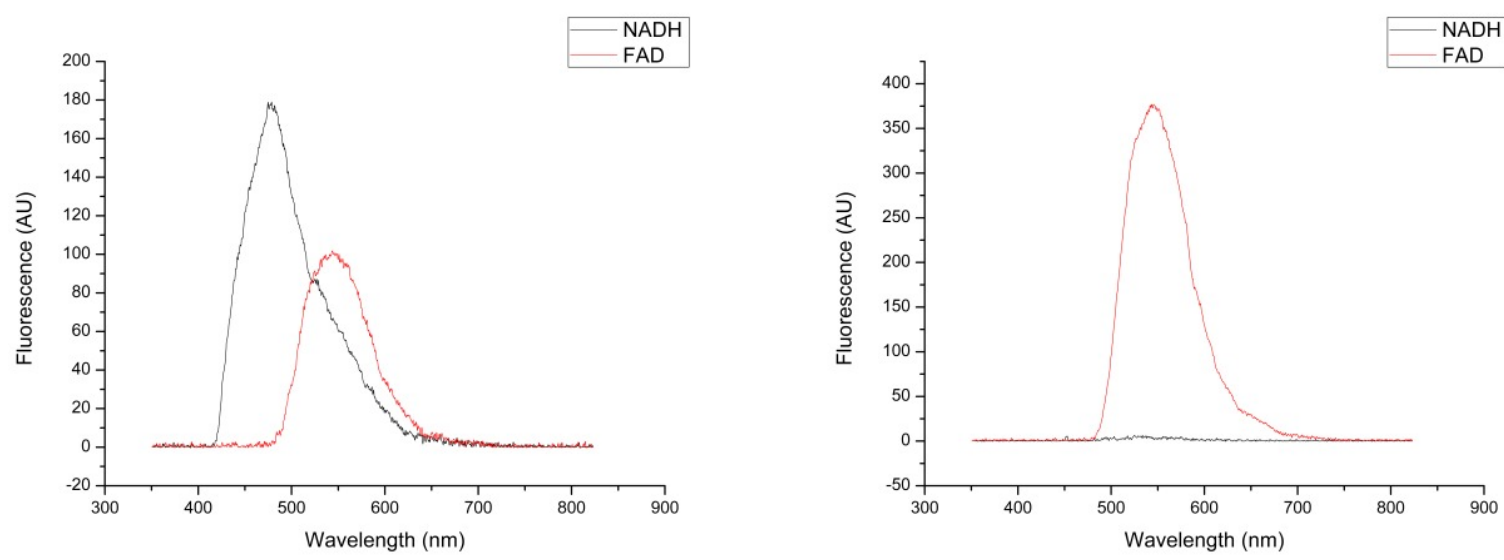

Fig.1: Fluorescence spectroscopy of NADH and FAD solutions (black line and red line, respectively) using 365nm excitation (a) and 450nm excitation (b)

\section{Confocal localisation of cellular autofluorescence}

Comparing cellular autofluorescence signals in Fig. 2 from NADH (Green image) and FAD (Purple image) over MitoTracker red signal (Red image) enables direct analysis of the localisation of autofluorescence. Figure 2 clearly shows that autofluorescence excited and recorded at the parameters used is arising from the mitochondria.
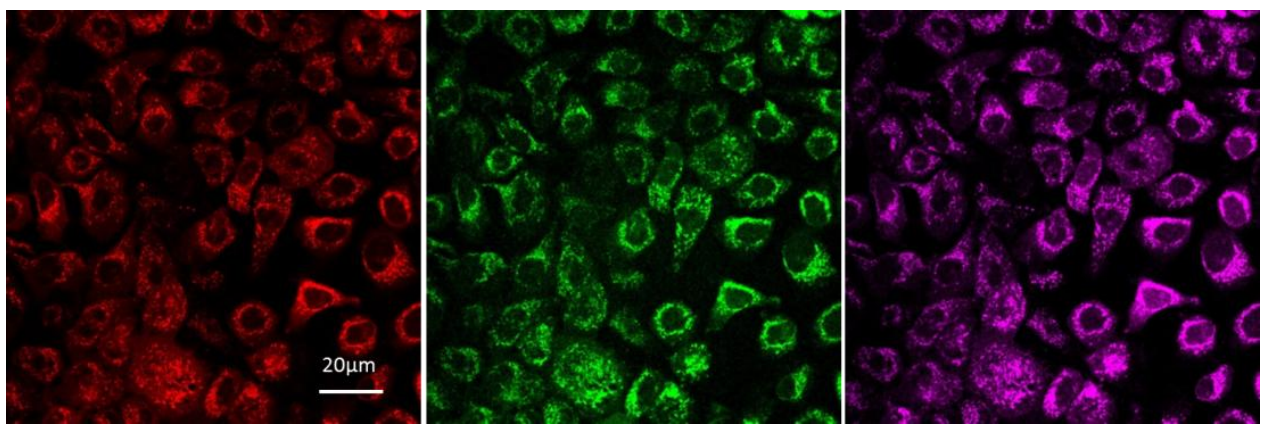

Fig.2: Confocal microscopy images of cellular localization of MitoTracker Red (red), NADH (green) and FAD (purple)

\section{Creation of "redox overview"}

Using NADH and FAD values for each cell, we can plot the metabolic profile of a population of cells, charting increasing NADH on the $\mathrm{x}$ axis and increasing FAD on the $\mathrm{y}$ axis. Fig. 3 displays the redox overviews of HUC healthy controls (a) and "5637" BCa cells (b) showing replicable differences in the population distributions. 

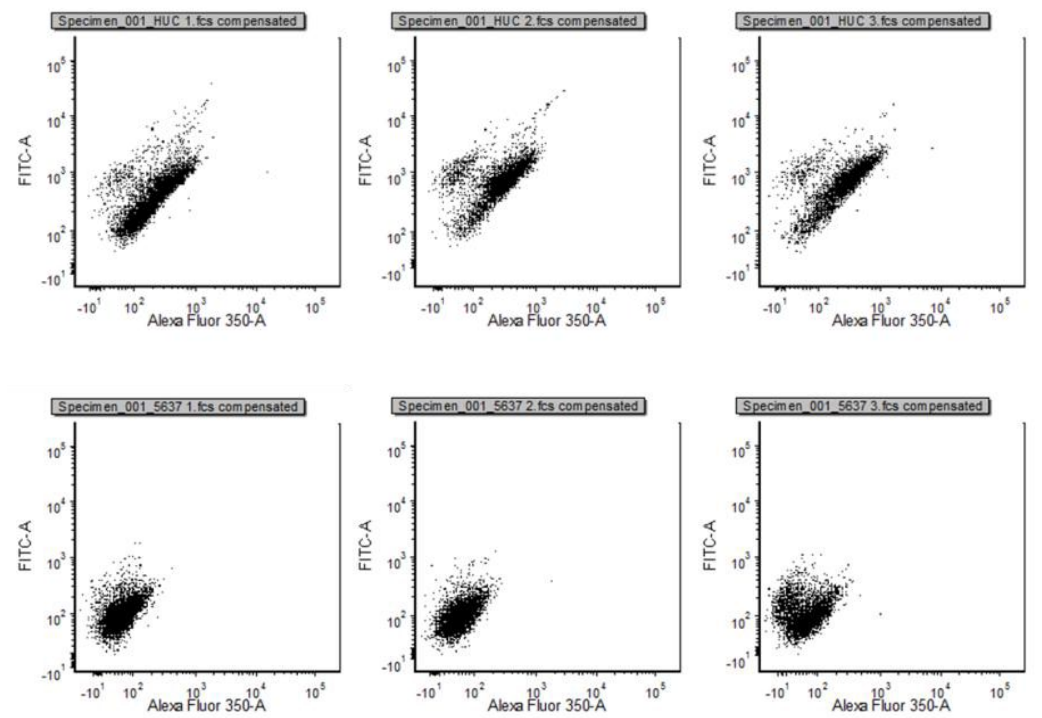

Fig.3: Redox overviews of (a) healthy urothelial cells and (b) 5637 BCa cells, charting increased NADH in x and increased FAD in y

\section{Comparison of average redox ratios of bladder cancer cells and healthy controls}

Fig.4 displays the mean and SEM optical redox ratios of healthy $(0.457+/-0.022)$ and cancer cells $(0.588+/-0.018)$. The $p$ value for these results, calculated using Student's t test, was found to be $<0.05$, allowing us to reject the Null hypothesis.

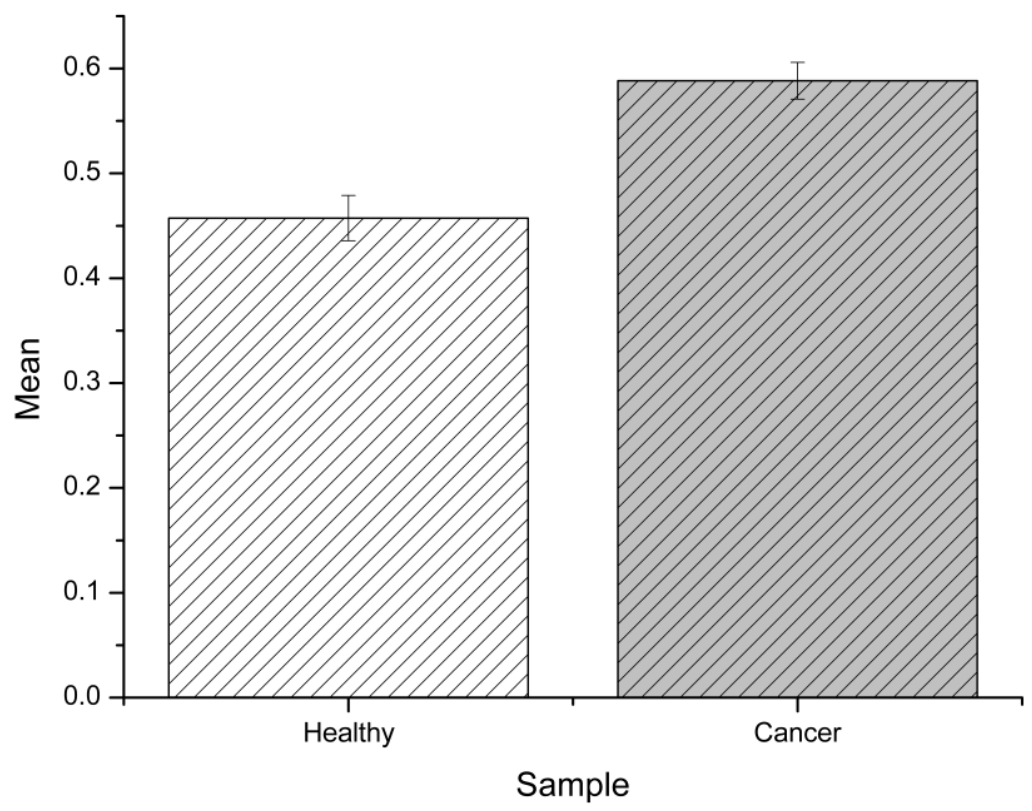

Fig.4: Comparison of the average redox ratios for three populations of healthy bladder and BCa cells, including SEM

Proc. of SPIE Vol. 9303 93030T-5 


\section{DISCUSSION}

We hypothesised that BCa cells would possess a significantly increased ORR compared to healthy controls owing to their selective accumulation of NADH. Statistical analysis of autofluorescence data has shown a $p<0.05$, confirming that the increased ORR observed in BCa cells is statistically significant. This finding helps to improve our understanding of the metabolic capabilities of healthy and cancerous bladder cells. BCa is therefore in keeping with other cancers, such as breast cancer ${ }^{18}$ in that it undergoes a metabolic switch resulting in mitochondrial accumulation of NADH. Importantly, we were capable of observing this difference using a conventional flow cytometer with customised band pass filters. Flow cytometry has a number of useful applications in clinical cancer diagnosis ${ }^{19}$ including DNA image cytometry for $\mathrm{BCa}^{20}$. Flow cytometry as a clinical adjunct for $\mathrm{BCa}$ detection and monitoring is therefore an established procedure, confirming sufficient cell yield in voided urine of $\mathrm{BCa}$ patients for comprehensive analysis. This technique presents some advantages over microscopy for clinical cellular analysis as cells can be rapidly processed and analysed and fluorescence quantified automatically. Autofluorescence flow cytometry is a poorly studied field, however the necessary removal of autofluorescence signals from conventional flow results ${ }^{21}$ suggests it warrants further investigation in its own right. The idea of NADH and FAD monitoring using flow cytometry was first suggested in the 1980 ' $\mathrm{s}^{22}$, but to our knowledge, ours is the first demonstration of the clinical worth of autofluorescence flow cytometry in bladder cancer monitoring. Autofluorescence flow cytometry of voided urine may serve to augment the sensitivity of conventional diagnostic tests, providing a cheap and rapid technique to aid in screening programmes which are currently hampered by time and $\cos ^{23}$. As demonstrated in this paper, the metabolic fluorophores NADH and FAD have defined excitation and emission parameters which allow their specification in flow setups. Rationing of NADH and FAD to create the ORR allows us to account for the spectral overlap in the fluorophores and also for size and granularity disparities in cells. The ORR is therefore a relatively reliable method for calculating the metabolic conditions of cells. The replicable differences in redox overview (NADH amplitude versus FAD amplitude) between healthy and cancer samples suggest diagnostic thresholding at this stage is a possibility. Diagnostic thresholding helps clinicians by giving automated diagnoses without the need for detailed interpretation, however this step would require validation from extensive patient studies. One of the major current limitations of flow cytometry to clinical applicability is the size and bulkiness of many benchtop analysers. Ongoing research is constantly reducing the size and complexity of flow cytometers and recently a mobile phone based single colour cytometer has been announced ${ }^{24}$. Size limitations of cytometry therefore may no longer be the issue they once were. Similarly, constant progress in laser diode development may help us overcome limitations with the relatively low quantum yield of endogenous fluorophores.

\section{CONCLUSION}

In this work we have demonstrated a statistically significant increase in the optical redox ratio (ORR) of bladder cancer cells compared to healthy cells, owing to selective mitochondrial accumulation of NADH in cancer cells which have undergone glycolytic switching. The application of the autofluorescence based flow cytometry method we have described could help to augment clinical work in voided urine cytology for diagnosis of bladder cancer.

\section{ACKNOWLEDGEMENTS}

This work was supported by the EU FP7 project ABLADE (Advanced Bladder cancer Diagnosis and Therapy) with further contribution from NHS and EPSRC. We acknowledge the help and support of Dr Sam Swift and Dr Alan Prescott, microscopy core facility, University of Dundee and Dr Rosemary Clark and Ms Arlene Whigham in the flow cytometry core facility, University of Dundee. 